\title{
Endobronchial ultrasound-guided cryobiopsies in peripheral pulmonary lesions: a feasibility study
}

\author{
Maren Schuhmann ${ }^{1,2}$, Korkut Bostanci ${ }^{1,3}$, António Bugalho ${ }^{4,5}$, Arne Warth ${ }^{6}$, \\ Philipp A. Schnabel ${ }^{7}$, Felix J.F. Herth ${ }^{1,2}$ and Ralf Eberhardt ${ }^{1,2}$
}

\begin{abstract}
Affiliations:
'Dept of Pulmonology and Critical Care Medicine, Thoraxklinik at the University of Heidelberg, Heidelberg, ${ }^{2}$ Translational Research Center Heidelberg, German Center for Lung Research (DZL), Heidelberg, ${ }^{6}$ Institute of Pathology, University of Heidelberg, Heidelberg, and

${ }^{7}$ Institute of Pathology, Section for Thoracic Pathology, University of Heidelberg. Heidelberg, Germany.

${ }^{3}$ Marmara University, Faculty of Medicine, Dept of Thoracic Surgery, Istanbul, Turkey.

4Interventional Pulmonology Unit, Hospital Beatriz Angelo, Loures, and

${ }^{5}$ Chronic Diseases Research Center (CEDOC), Faculdade de Ciências Médicas, Universidade Nova de Lisboa, Lisbon, Portugal.
\end{abstract}

Correspondence: M. Schuhmann, Pulmonology and Critical Care Medicine, Thoraxklinik at the University of Heidelberg, Amalienstr. 5, 69126 Heidelberg, Germany. E-mail: maren.schuhmannamed.uni-heidelberg.de

ABSTRACT Peripheral lung lesions are sometimes difficult to reach even with endobronchial ultrasound (EBUS) and insufficient material is often obtained by transbronchial forceps biopsy. Cryoprobes can be used for performing tissue biopsies. We evaluated the safety and feasibility of the cryoprobe in combination with EBUS for the diagnosis of peripheral lung lesion.

Patients with peripheral lung lesions of up to $4 \mathrm{~cm}$ were enrolled. After identifying the lung lesion by radial EBUS, forceps biopsies and cryobiopsies were performed in a randomised order. We evaluated safety and feasibility, and compared diagnostic yield and sample size.

39 patients were randomised and the peripheral lung lesion was reached in 31 . The overall diagnostic yield was $60.5 \%$ and, in the lesions reached by EBUS, it was $74.2 \%$. In 19 cases, the diagnosis was made with forceps as well as cryobiopsy and, in four cases, only with cryobiopsy. Cryobiopsies were significantly larger than forceps biopsies $\left(11.17 \mathrm{~mm}^{2}\right.$ versus $\left.4.69 \mathrm{~mm}^{2}, \mathrm{p}<0.001\right)$. We observed one case of moderate bleeding.

Transbronchial cryobiopsy with EBUS guidance is safe and useful to obtain histological samples. Larger tissue samples can be obtained by cryoprobe.

@ERSpublications

Transbronchial cryobiopsy with EBUS-guidance is safe and useful to obtain samples from peripheral lesions http://ow.ly/pPry9 


\section{Introduction}

Lung cancer is one of the main causes of cancer-related death worldwide [1]. When detected in the early stages, curative surgical options can be considered, improving survival significantly. Screening for lung cancer in order to detect the tumour earlier is frequently discussed, and recently the National Lung Screening Trial was able to show an improvement in mortality data in patients screened on a yearly basis with low-dose computed tomography (CT) [2]. At the same time, however, there was an increase of incidentally detected solitary pulmonary lesions of unknown "histology".

In order to obtain a definitive diagnosis of these lesions it is often mandatory to perform tissue sampling. Transthoracic CT-guided needle biopsy can be performed, but has a high rate of pneumothorax quoted to be between $15 \%$ and $43 \%$, with between $4 \%$ and $18 \%$ requiring a chest drain, as well as the risk of pulmonary haemorrhage with reported frequencies of between $1.0 \%$ and $27 \%[3,4]$. Transbronchial forceps biopsy can be performed in combination with other techniques, such as brushing, needle biopsy or washings [5]. The sensitivity is quite variable and depends upon the size of the lesion as well as the distance to the pleura $[5,6]$.

The use of guidance techniques, such as endobronchial ultrasound (EBUS), virtual bronchoscopy and electromagnetic navigated bronchoscopy (ENB), improves the diagnostic yield to $\sim 70 \%$, although the sensitivity depends strongly on the biopsy technique [7-12]. Biopsies obtained with these conventional techniques are often small and may not be suitable for further immunohistochemical or molecular studies [13]. Therefore the biopsy technique has to be improved further.

The cryoprobe has been used within the airway for tumour destruction by freezing and thawing. In recent years, cryoprobes have been established for airway recanalisation and for sampling endobronchial lesions, particularly in patients with lung cancer [14-16]. Due to the simplicity of handling and the larger biopsy sizes, first reports about the use of cryoprobes for transbronchial biopsies have been published [17, 18]. However, in all of these trials, this technique has been used in patients with diffuse or interstitial lung disease.

We assessed the safety, feasibility and efficacy of the cryoprobe for transbronchial biopsies in solitary pulmonary lesions with the guidance of radial endobronchial ultrasound.

\section{Patients and methods}

Between March 2010 and September 2010, 39 consecutive patients with a solid pulmonary lesion of $<40 \mathrm{~mm}$, who were referred to our institution for diagnostic bronchoscopy, were included in the study. All patients had a CT scan performed prior to bronchoscopy and lesion size was measured at its largest diameter. All patients were included irrespective of the position of the lesion and the relationship of the lesion to the airway. Patients with tumours suspicious of endobronchial growth, pure ground-glass lesions, mediastinal lymphadenopathy or contraindications for bronchoscopy and transbronchial biopsies were excluded. The study protocol had been approved by the ethics committee of the University of Heidelberg (S320/2009) and the study design was registered at the US National Institutes of Health (NCT01221493). All patients signed a study consent form.

In all 39 cases, a combined rigid and flexible bronchoscopy was performed according to our standard protocol at Thoraxklinik Heidelberg, Heidelberg, Germany, by three trainees under the supervision of an experienced bronchoscopist. First, the central airways were assessed endoscopically. If endobronchial tumour growth was visible, the patient was excluded from the study. Based on the CT of the thorax, a radial EBUS probe (UM-S20-20R; Olympus, Tokyo, Japan) with flexible guide-sheath was advanced into the suspected bronchial segments to detect the peripheral lesion. This navigation technique has been described previously in other publications [19-21]. Additional fluoroscopy was used if this was considered necessary by the investigator. The duration of EBUS use for the detection of the lesion was limited to $20 \mathrm{~min}$. If the lesion was not detected within the given time, the patient was considered as EBUS negative and excluded from the study.

Once the lesion was detected by EBUS, the position of the probe in relation to the lesion was noted. The miniprobe was removed whilst the guide sheath remained in position. All patients received three transbronchial biopsies of their lung lesion with forceps as well as three with the cryoprobe. Patients were randomised to receive either the forceps or the cryobiopsies first according to a randomisation list with a distribution of 1:1 (fig. 1).

We used a commercially available reusable forceps (FB-19-C $120 \mathrm{~cm}$; Olympus) through the guide sheath. The correct position of the guide sheath was confirmed at the end of the three biopsies by EBUS to exclude dislocation. 
FIGURE 1 Flow diagram of endobronchial ultrasound (EBUS) and biopsy procedures performed.

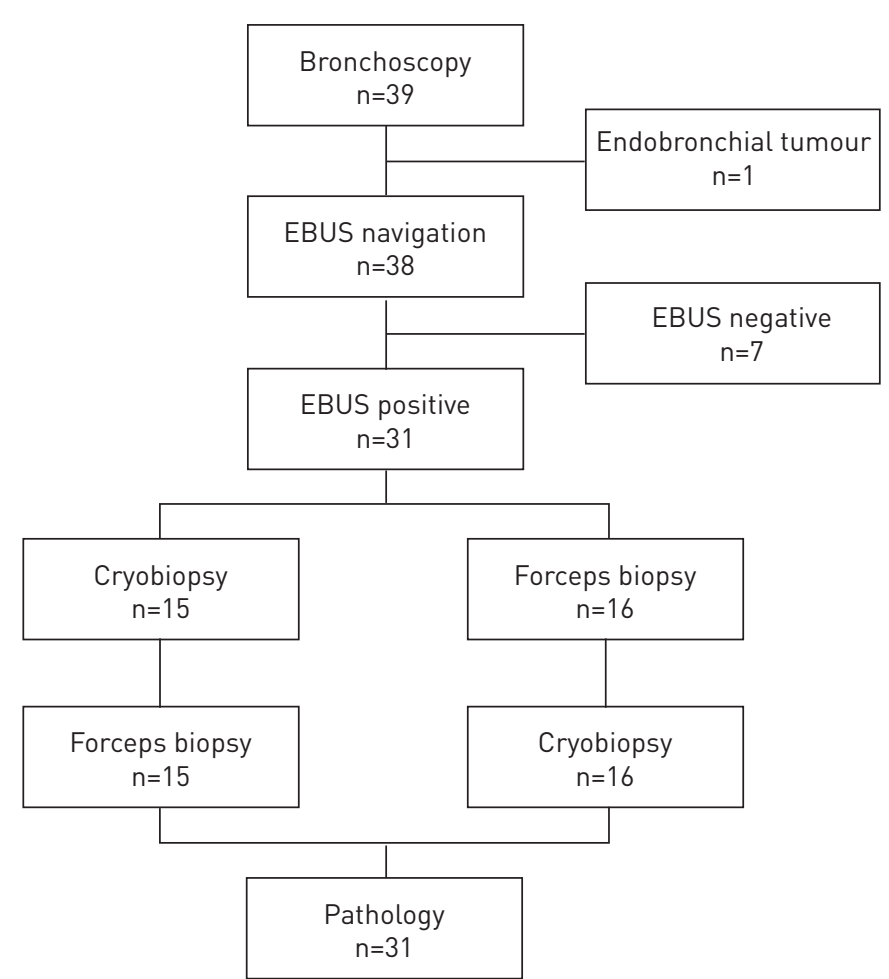

For the cryobiopsies, a flexible cryoprobe of $1.2 \mathrm{~mm}(1.7 \mathrm{~mm}$ with oversheath) diameter and $90 \mathrm{~cm}$ length (ERBE, Solingen, Germany) was used. The tip of the probe was cooled with nitrous oxide to a temperature of $-89^{\circ} \mathrm{C}$. The probe was cooled for $\sim 4 \mathrm{~s}$ and immediately thereafter retracted together with the guide sheath and bronchoscope. The frozen biopsy was then thawed in normal saline and fixed in formalin. After each cryobiopsy, the lesion was located again by radial ultrasound and fluoroscopy if required. After six biopsies, the examination was terminated and, $2 \mathrm{~h}$ after the procedure, a chest radiograph was performed.

All samples were fixed separately in formalin and processed in the Institute of Pathology at the University of Heidelberg, Heidelberg, Germany. The pathologists were blinded to the biopsy technique and to the order of the biopsy. The samples were embedded in paraffin and then mounted onto glass slides. Haematoxylin and eosin and periodic acid-Schiff stainings were performed. First, a histological diagnosis was established. If transbronchial biopsy yielded a definitive histological diagnosis, it was considered to be a transbronchial biopsy success. If the transbronchial biopsy result was nondiagnostic, then additional procedures, such as CT-guided transthoracic needle biopsy or surgery, were undertaken to confirm the diagnosis. However, if transbronchial biopsy yielded a plausible benign diagnosis, the patient was treated accordingly or observed for 2 years to exclude malignancy.

The length of time required for the detection of the lesion as well as for the individual biopsy techniques was recorded. Bleeding and complications, such as postinterventional pneumothorax, as well as histological outcomes, were documented.

A biopsy was assumed as diagnostic overall if at least one of the three biopsies obtained with the cryobiopsy or forceps technique was diagnostic. Diagnostic yield was calculated for each biopsy technique as the number of diagnostic procedures divided by the number of nondiagnostic procedures plus number of diagnostic procedures.

All samples underwent planimetric assessment by measuring the area of the histological specimen with a digital system (SteREO Discovery.V12 and the AxioVision 4.5 software; Carl Zeiss Microscopy GmbH, Göttingen, Germany).

The artefact-free specimen area was assessed by a pathologist, blinded to the biopsy technique, by applying a semiquantitative scoring scale divided into five equal steps $(0-20,21-40,41-60,61-80$ and $81-100 \%)$ describing the relative amount of unaltered tissue.

Data were analysed by means of descriptive statistics (means and standard deviations), as well as by statistical hypothesis testing. For non-normally distributed samples, a nonparametric test (Wilcoxon 
matched pairs signed rank test) was used. Fisher's exact test was used to compare proportions. All p-values ( $\mathrm{p}<0.05$ was considered statistically significant) are two-sided and were not adjusted for the number of parameters evaluated. Statistical analysis was performed on a computer using PRISM 6.0 software (GraphPad Software, Inc., La Jolla, CA, USA).

\section{Results}

39 patients (28 male and 11 female) were included in this prospective study. The mean age was 68 years (range 37-84 years). One patient was excluded due to visible endobronchial tumour. The remaining 38 patients had a lung lesion of $29.7 \pm 7.3 \mathrm{~mm}$ in diameter, of which 31 were malignant and seven were benign. On average, the distance to the pleura was $7 \pm 10 \mathrm{~mm}$. The distribution of the lesions is described in detail in table 1. The average time for EBUS examination was $7.6 \pm 6.1 \mathrm{~min}$. The EBUS detection rate was $81.6 \%$. We were not able to locate the tumour after $20 \mathrm{~min}$ in seven of the 38 patients, and these patients did not receive transbronchial biopsy. The detection rate was independent of the location of the lesion except for the middle lobe, where all lesions could be detected by EBUS.

In the remaining 31 patients, the EBUS probe was located within the lesion in $20(64.5 \%)$ cases while, in 11 $(35.5 \%)$ cases, the EBUS probe was located adjacent to the lesion.

All 31 EBUS-positive patients received three transbronchial forceps biopsies and three transbronchial cryobiopsies according to the study protocol. The average time required for forceps biopsy was significantly shorter than the time required for the cryobiopsies $(5.1 \pm 2.75 \mathrm{~min}$ and $11.6 \pm 4.4 \mathrm{~min}$, respectively; $\mathrm{p}<0.0001)$.

The overall diagnostic yield, including lesions not biopsied as we were unable to detect them by EBUS, was $60.5 \%$ ( 23 out of 38 patients). The diagnostic yield of the lesions that we were able to visualise with EBUS was $74.2 \%$ (23 out of 31 patients). In 19 cases, both techniques established a diagnosis. Additionally, four cases that were nondiagnostic with forceps biopsy were successfully diagnosed with cryobiopsy resulting in a diagnostic yield of $61.3 \%$ (19 out of 31 ) for forceps and $74.2 \%$ (23 out of 31 ) for cryobiopsy, respectively $(\mathrm{p}=0.42)$. Taking only the first biopsy into account, $19(61.3 \%)$ out of 31 patients were diagnosed by cryobiopsy and $15(48.4 \%)$ by forceps biopsy $(\mathrm{p}=0.44)$. The cumulative yield after the second biopsy was $71 \%$ ( 22 out of 31 patients) with the cryoprobe and $54.9 \%$ (17 out of 31 ) with forceps; however, the difference in yield between both techniques was not significant $(\mathrm{p}=0.29)$ (fig. 2). It should be noted that, in those four cases where only cryobiopsy was successful in securing a diagnosis, no differences were observed in the positioning of the EBUS probe or in the size of the lesion and histological assessment. The histology of these four cases was two adenocarcinomas, one squamous cell carcinoma and one hamartoma.

The results of both biopsy techniques overall and independent of the final histological diagnosis are summarised in table 2.

Of the 93 biopsies taken for each technique in total, $54(58.1 \%)$ of the cryobiopsies and $43(46.2 \%)$ of the forceps biopsies were diagnostic $(\mathrm{p}=0.14)$.

Quantitative digital assessment of histological samples showed a mean sample area of $11.17 \mathrm{~mm}^{2}$ (range 1.25$38.59 \mathrm{~mm}^{2}$ ) for cryobiopsy and $4.69 \mathrm{~mm}^{2}$ (range $0.53-22 \mathrm{~mm}^{2}$ ) for forceps biopsy $(\mathrm{p}<0.001)$. No qualitative

TABLE 1 Endobronchial ultrasound (EBUS) results in relation to distribution of the lesion using Fisher's exact test

\begin{tabular}{lccccc} 
Lobes & Position & Total & EBUS positive & EBUS negative & p-values \\
\hline \multirow{2}{*}{ Upper } & $\mathrm{R}$ & 13 & $11(84.6)$ & $2(15.4)$ & 1 \\
& $\mathrm{~L}$ & 8 & $6(75.0)$ & $2(25.0)$ & 0.64 \\
\multirow{3}{*}{ Middle } & Total & 21 & $17(81.0)$ & $4(19.0)$ & 1 \\
Lower & $\mathrm{R}$ & 5 & $5(100.0)$ & $0(0.0)$ & 0.57 \\
& $\mathrm{R}$ & 7 & $5(71.4)$ & $2(19.6)$ & 0.61 \\
\multirow{3}{*}{ All } & $\mathrm{L}$ & 5 & $4(80.0)$ & $1(20.0)$ & 1 \\
& Total & 12 & $9(75.0)$ & $3(25.0)$ & 0.69 \\
& $\mathrm{R}$ & 25 & $21(84.0)$ & $4(16.0)$ & 1 \\
& $\mathrm{~L}$ & 13 & $10(76.9)$ & $3(23.1)$ & 0.7 \\
\hline
\end{tabular}

Data are presented as $\mathrm{n}$ or $\mathrm{n}(\%)$, unless otherwise stated. L: left; R: right. 
FIGURE 2 The cumulative diagnostic yield following cryobiopsy and biopsy with forceps, shown as the number of diagnostic specimens obtained after the first biopsy, the cumulative number of diagnostic specimens after the first and second biopsies and after all three biopsies.

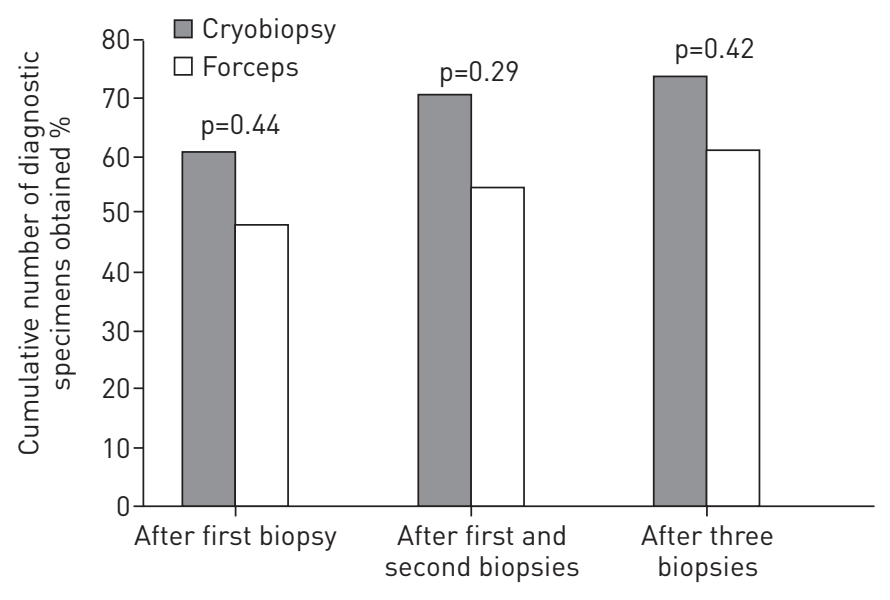

difference was observed between cryobiopsy and forceps biopsy. Retrieval of biopsies with cryoprobe did not result in more artefacts compared with forceps biopsy. Applying the semiquantitative scoring of tissue morphology, 93 samples of the cryobiopsies and 91 samples of the forceps biopsy showed $>80 \%$ unaltered relative tissue area.

No severe complications were observed during this study. There was one case of moderate bleeding at the end of all six biopsies necessitating prolonged suction with the bronchoscope, but no other intervention. No pneumothorax was detected on chest radiography.

\section{Discussion}

Peripheral lung lesions still pose a diagnostic dilemma. One challenge is to improve the biopsy techniques that are currently available. A recent study showed that the use of cryoprobes for diagnosing endobronchial malignancy was superior to forceps biopsies [14]. Cryoprobes have also previously been used to perform transbronchial biopsies in interstitial lung disease [17].

In this study, we were able to show, for the first time, that the use of a cryoprobe for transbronchial biopsies in the diagnosis of pulmonary lesions is feasible.

We combined this technique with the use of radial endobronchial ultrasound and a guide sheath in order to improve the rate of detection. In this trial, our detection rate for pulmonary lesions was $81.6 \%$ with EBUS, which correlates with values described in the literature [22]. In seven out of 38 patients, we were not able to locate the lesion using EBUS within $20 \mathrm{~min}$ and these patients were therefore excluded from our study. This exclusion was necessary because the probability of a positive diagnosis in EBUS-negative lesions is reported to be very low [20]. In all 31 patients in whom we detected the lesion by EBUS, we were able to perform forceps biopsies and cryobiopsies and adequate specimens were obtained.

\section{TABLE 2 Histological diagnosis of endobronchial ultrasound-positive lesions using Fisher's exact test}

\begin{tabular}{|c|c|c|c|c|c|}
\hline Diagnosis & Lesions & Cryobiopsy & Forceps biopsy & Total yield & p-value \\
\hline \multicolumn{6}{|l|}{ Malignant } \\
\hline Squamous cell & 7 & $5(71.4)$ & $4(57.1)$ & $5(71.4)$ & 1 \\
\hline Carcinoma & 5 & $3(60.0)$ & $3(60.0)$ & $3(60.0)$ & 1 \\
\hline Total & 25 & 17 (68.0) & $14(0.56)$ & 17 (68.0) & 0.76 \\
\hline \multicolumn{6}{|l|}{ Benign } \\
\hline Hamartoma & 1 & $1(100)$ & $0(0)$ & $1(100)$ & 1 \\
\hline Granuloma & 1 & $1(100)$ & $1(100)$ & $1(100)$ & 1 \\
\hline Total & 6 & $6(100)$ & 5 (83.3) & $6(100)$ & 1 \\
\hline Total lesions & 31 & 23 (74.2) & 19 (61.3) & 23 (74.2) & 0.42 \\
\hline
\end{tabular}

Data are presented as $\mathrm{n}$ or $\mathrm{n}(\%)$, unless otherwise stated. COP: cryptogenic organising pneumonia. 
The duration of the cryobiopsies was significantly longer in comparison with forceps biopsy. The longer time was due to the need to remove the guide sheath for each biopsy, requiring repeat localisation of the lesion with EBUS prior to repeat sampling. A prolonged procedure time may be acceptable in order to obtain larger samples. This might reduce the number of biopsies that need to be performed, overcoming this concern in the future.

Relevant potential complications for transbronchial forceps biopsy are iatrogenic bleeding and pneumothorax. The rates for pneumothorax after EBUS-guided transbronchial forceps biopsies are reported to be $0-5.1 \%$ [7]. BABIAK et al. [17] quoted a pneumothorax incidence of nearly 5\% after transbronchial biopsies with a cryoprobe in interstitial lung disease. We did not observe any pneumothorax in our study. This may be due to the fact that we only obtained biopsies in patients in whom the lesion was reached by EBUS prior to performing the biopsy and in whom we additionally controlled the position of the sheath and the biopsy instruments with fluoroscopy; biopsy or damage of the pleura is hence minimised. Ideally, five biopsies should be taken to optimise the diagnostic yield $[11,20]$, although the risk of bleeding increases with the number of biopsies taken [23]. Due to the crossover design of the study, we took six biopsies in every patient. In the study performed by HETZEL et al. [14], an increased incidence of bleeding after endobronchial cryobiopsies was observed in comparison with forceps biopsies, but the number of significant haemorrhages requiring intervention did not differ compared with forceps biopsy. In our study, we only had one moderate bleed that required no further procedures other than suction. Due to the combined use of both biopsy techniques in every patient, we could not differentiate the safety data and assign them to one of the techniques. However, the number of complications in our study was very low and, therefore, the use of cryobiopsies for peripheral lung lesions appears to be safe. Again, this may also be due to the combined use of EBUS and guide sheath detection of the lesion prior to sampling.

Even when the lesion is detected using EBUS, a certain amount of biopsies are nondiagnostic [20, 22, 24]. This is the result of a sampling error, either due to the position of the EBUS probe in relation to the lesion or due to dislocation of the biopsy tool, which can be overcome by the use of concurrent fluoroscopy. As reported in the literature, the diagnostic yield increases up to $>80 \%$ when the miniprobe can be positioned centrally within the lesion rather than adjacent to the lesion, which has a yield of only $44-61 \%[20,22,24]$. The diagnostic yield in all lesions in our study was $60.5 \%$. The diagnostic yield in the EBUS-positive lesions in our study was $74.2 \%$ ( 23 out of 31 patients). The distribution of diagnostic yield in relation to the position of the EBUS probe, either within or adjacent to the lesion, was similar to the published literature ( $85 \%$ versus $54.5 \%$, respectively; $\mathrm{p}=0.09$ ). However, in the seven EBUS-negative cases, we were not allowed to take any biopsies according to the study protocol.

A better diagnostic yield, as well as a higher number of diagnostic biopsies, was shown for the cryoprobe, but this was not statistically significant. The biopsy tools follow the path of the bronchus and, if the bronchus is only adjacent to the tumour, forceps sampling often misses the lesion. When using a cryoprobe, the tissue surrounding the tip is frozen, thus also enabling biopsies in a lateral direction. This might be one of the major advantages, especially in lesions where EBUS cannot be placed within the lesion. The position of the EBUS probe in those four cases where only the cryoprobe was diagnostic was within as well as adjacent to the lesion.

Another advantage of the cryoprobe may be the larger samples obtained with the procedure, supplying the pathologist with more tissue from which to make a correct diagnosis and to perform further molecular analyses, which are crucial to tailoring lung cancer treatment [13]. In our study, the size of the samples obtained with the cryoprobe was approximately three times larger than those acquired with conventional forceps, as has been reported in previous trials $[14,17,18]$.

Cryoprobe samples tend to have larger artefact-free areas in comparison with forceps samples, although this trend could not be shown in our study $[14,17]$. The combination of different biopsy tools usually leads to a higher diagnostic yield. In our study, forceps biopsies did not add to the yield of cryobiopsies.

This study was designed to show the feasibility and safety of cryosampling in patients with peripheral lesions. It was therefore not powered to show superiority in comparison with forceps biopsy. Safety should also be confirmed in a larger trial. Another limitation of this trial is the crossover design using both techniques in every patient. Because this was the first use of the cryoprobe in peripheral lung lesions, excluding patients from the standard approach of forceps biopsy was not possible.

In conclusion, the use of cryoprobes for obtaining biopsies from peripheral lung lesions is feasible. In comparison with forceps biopsy, significantly larger samples can be obtained without affecting safety. In order to show the possible advantages of the cryoprobe, such as a potentially higher diagnostic yield, further larger trials in a randomised setting need to be performed. 


\section{References}

1 Bach PB, Silvestri GA, Hanger M, et al. Screening for lung cancer: ACCP evidence-based clinical practice guidelines (2nd edition). Chest 2007; 132: Suppl., 69S-77S.

2 Aberle DR, Adams AM, Berg CD, et al. Reduced lung-cancer mortality with low-dose computed tomographic screening. N Engl J Med 2011; 365: 395-409.

3 Khan MF, Straub R, Moghaddam SR, et al. Variables affecting the risk of pneumothorax and intrapulmonal hemorrhage in CT-guided transthoracic biopsy. Eur Radiol 2008; 18: 1356-1363.

4 Wiener RS, Schwartz LM, Woloshin S, et al. Population-based risk for complications after transthoracic needle lung biopsy of a pulmonary nodule: an analysis of discharge records. Ann Intern Med 2011; 155: 137-144.

5 Gasparini S. In: Strausz J, Bolliger CT, eds. Diagnostic management of solitary pulmonary nodule. Eur Respir Monogr 2010; 48: 90-108.

6 Schreiber G, McCrory DC. Performance characteristics of different modalities for diagnosis of suspected lung cancer: summary of published evidence. Chest 2003; 123: Suppl., 115S-128S.

7 Steinfort DP, Khor YH, Manser RL, et al. Radial probe endobronchial ultrasound for the diagnosis of peripheral lung cancer: systematic review and meta-analysis. Eur Respir J 2011; 37: 902-910.

8 Eberhardt R, Anantham D, Ernst A, et al. Multimodality bronchoscopic diagnosis of peripheral lung lesions: a randomized controlled trial. Am J Respir Crit Care Med 2007; 176: 36-41.

9 Oki M, Saka H, Kitagawa C, et al. Randomized study of endobronchial ultrasound-guided transbronchial biopsy: thin bronchoscopic method versus guide sheath method. J Thorac Oncol 2012; 7: 535-541.

10 Eberhardt R, Kahn N, Gompelmann D, et al. LungPoint - a new approach to peripheral lesions. J Thorac Oncol 2010; 5: 1559-1563.

11 Asahina H, Yamazaki K, Onodera Y, et al. Transbronchial biopsy using endobronchial ultrasonography with a guide sheath and virtual bronchoscopic navigation. Chest 2005; 128: 1761-1765.

12 Wang Memoli JS, Nietert PJ, Silvestri GA. Meta-analysis of guided bronchoscopy for the evaluation of the pulmonary nodule. Chest 2012; 142: 385-393.

13 Travis WD, Brambilla E, Noguchi M, et al. Diagnosis of lung cancer in small biopsies and cytology: implications of the 2011 International Association for the Study of Lung Cancer/American Thoracic Society/European Respiratory Society Classification. Arch Pathol Lab Med 2013; 137: 668-684.

14 Hetzel J, Eberhardt R, Herth FJ, et al. Cryobiopsy increases the diagnostic yield of endobronchial biopsy: a multicentre trial. Eur Respir J 2012; 39: 685-690.

15 Schumann C, Hetzel M, Babiak AJ, et al. Endobronchial tumor debulking with a flexible cryoprobe for immediate treatment of malignant stenosis. J Thorac Cardiovasc Surg 2010; 139: 997-1000.

16 Hetzel M, Hetzel J, Schumann C, et al. Cryorecanalization: a new approach for the immediate management of acute airway obstruction. J Thorac Cardiovasc Surg 2004; 127: 1427-1431.

17 Babiak A, Hetzel J, Krishna G, et al. Transbronchial cryobiopsy: a new tool for lung biopsies. Respiration 2009; 78: 203-208.

18 Griff S, Ammenwerth W, Schonfeld N, et al. Morphometrical analysis of transbronchial cryobiopsies. Diagn Pathol 2011; 6: 53.

19 Kurimoto N, Miyazawa T, Okimasa S, et al. Endobronchial ultrasonography using a guide sheath increases the ability to diagnose peripheral pulmonary lesions endoscopically. Chest 2004; 126: 959-965.

20 Yamada N, Yamazaki K, Kurimoto N, et al. Factors related to diagnostic yield of transbronchial biopsy using endobronchial ultrasonography with a guide sheath in small peripheral pulmonary lesions. Chest 2007; 132: 603-608.

21 Yoshikawa M, Sukoh N, Yamazaki K, et al. Diagnostic value of endobronchial ultrasonography with a guide sheath for peripheral pulmonary lesions without X-ray fluoroscopy. Chest 2007; 131: 1788-1793.

22 Huang CT, Ho CC, Tsai YJ, et al. Factors influencing visibility and diagnostic yield of transbronchial biopsy using endobronchial ultrasound in peripheral pulmonary lesions. Respirology 2009; 14: 859-864.

23 Hopkins PM, Aboyoun CL, Chhajed PN, et al. Prospective analysis of 1,235 transbronchial lung biopsies in lung transplant recipients. J Heart Lung Transplant 2002; 21: 1062-1067.

24 Chao TY, Chien MT, Lie CH, et al. Endobronchial ultrasonography-guided transbronchial needle aspiration increases the diagnostic yield of peripheral pulmonary lesions: a randomized trial. Chest 2009; 136: 229-236. 